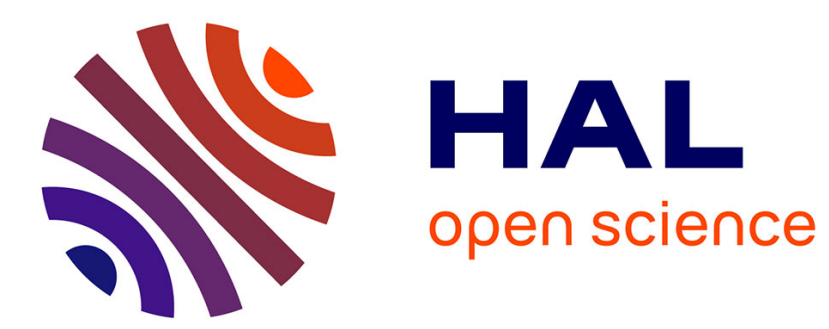

\title{
Genèse idéologique d'une réforme
}

\author{
Danièle Lochak
}

\section{To cite this version:}

Danièle Lochak. Genèse idéologique d'une réforme. Hommes \& migrations, 1994, Les lois "Pasqua", 1178, pp.23- 29. hal-01674316

\section{HAL Id: hal-01674316 https://hal.parisnanterre.fr/hal-01674316}

Submitted on 2 Jan 2018

HAL is a multi-disciplinary open access archive for the deposit and dissemination of scientific research documents, whether they are published or not. The documents may come from teaching and research institutions in France or abroad, or from public or private research centers.
L'archive ouverte pluridisciplinaire HAL, est destinée au dépôt et à la diffusion de documents scientifiques de niveau recherche, publiés ou non, émanant des établissements d'enseignement et de recherche français ou étrangers, des laboratoires publics ou privés. 


\section{GENÈSE IDÉOLOGIQUE Pour Daniele
Lochak. l'objectif de}

la loi du 22 juillet 1993 est clair : presque toutes les modifications à la législation en vigueur ont pour objet de rendre plus difficile l'accès à la nationalité francaise. Mais le plus inquiétant, selon elle, c'est la justification idéologique de cette réforme : l'infusion à trop haute dose d'étrangers dans la population française remettrait en cause l'identité

par Danièle LOCHAK

Professeur

à Paris X-Nanterre. Présidente du GISTI.
L' État-nation, dans la tradition républicaine française, se caractérise par un mélange de fermeiure et d'ouverture : fermeture, puisque l'étranger, I| es) clu de la communauté nationale, est exclu simultanément de la communauté politique qu'elle forme ; Ouverture, cependant, dans la mesure où l'accès à la Thationalité française est toujours resté, avec des @yariations selon les époques, relativement aisé. La Révolution a ouvert largement l'accès à la nationaGité, ou plutôt à la citoyenneté française, en accordant une place égale à un droit du sol largement conçu et à la filiation. Et si, par la suite, le code civil est revenu au jus sanguinis, rendant ainsi plus étanche la frontière entre nationaux et étrangers, toute l'évolution ultérieure a été dans le sens d'une ouverture de plus en plus large de l'accès à la nationalité française : la loi de 1851 déclare français l'enfant né en France d'un étranger qui lui-même y est né, la loi de 1889 incorpore à la communauté nationale tous les individus présumés assimilables puisque nés en France, en décidant qu'ils deviennent français à leur majorité. Lois de circonstances, certes, pour une part, dictées par l'affaiblissement de la natalité française et les besoins du recrutement de l'armée ; mais dictées aussi par la préoccupation de maintenir l'égalité entre les Français, soumis à la conscription, et les immigrés de seconde génération qui, en restant étrangers, en seraient exemptés ; dictées enfin, plus fondamentalement encore, par le souci de ne pas voir se maintenir sur le territoire français des communautés étrangères soudées, susceptibles de menacer l'unité du pays. Ces lois ont imprimé durablement leur marque au droit de la nationalité et lui ont conféré son visage actuel, expression d'une conception assimilationniste de la nation et d'une conception pragmatique, plus qu'idéologique, du droit.

C'est ce système ouvert, et enraciné en France depuis près d'un siècle, que l'extrême droite, bientôt suivie par la droite, a entrepris de contester à partir de 1985. Invoquant la menace qu'une population d'origine étrangère trop nombreuse ferait peser sur l'identité nationale, cette même droite va réclamer, et finalement obtenir, une réforme du code de la nationalité rendant moins aisé l'accès à la nationalité française.

Contrairement à ce qui avait été le cas pendant l'entre-deux-guerres, où le droit de la nationalité avait déjà donné lieu à de très vifs débats, l'essentiel des critiques ne porte pas cette fois sur la procédure de naturalisation mais sur l'attribution de la nationalité française par l'effet du jus soli : l'automaticité du système en vigueur aboutirait à accorder la nationalité française à des personnes qui ne le souhaitent pas réellement ou qui n'ont pas conscience d'être devenues françaises.

La démarche est habile car la critique est séduisante : comment ne pas souscrire à l'idée que la nationalité devrait résulter d'une adhésion consciente et volontaire et non d'un choix imposé ? Mais, derrière l'argument apparemment rationnel du refus de l'automaticité, il y a en réalité un implicite idéologiquement beaucoup plus douteux : l'idée que l'infusion à trop haute dose d'étrangers - et qui plus est d'étrangers non européens - dans la population française remettrait en cause l'identité nationale.

L'automaticité, au demeurant, n'est le monopole ni de la législation française, ni de l'application du jus soli : en règle générale, on ne choisit pas sa nationalité ; on est national ou étranger selon une 
détermination strictement juridique laissée pour l'essentiel à l'appréciation de l'État, et dans laquelle les aspirations individuelles de chacun n'ont qu'une part limitée.

Les griefs articulés contre le jus soli ne sont pas plus fondés. Le fait d'être né et d'avoir vécu dans un pays crée, à l'évidence, des liens potentiellement aussi forts avec ce pays que la circonstance d'être né d'un père ou d'une mère qui en a la nationalité. Or, l'insistance mise sur la nécessité d'un acte volontaire d'adhésion pour devenir français laisse implicitement entendre que la naissance en France, même complétée par la résidence, ne suffit pas à faire de vrais Français, puisqu'à aucun moment on n'envisage d'imposer cet acte volontaire à ceux qui sont nés, y compris à l'étranger, de parents - ou même d'un seul parent - français. Autrement dit, le sentiment d'appartenance nationale se transmettrait plus sûrement par les gènes que par l'École de la République.

Le projet de réforme du code de la nationalité déposé par le ministre de la Justice devant l'Assemblée nationale à l'automne 1986, pendant la période de cohabitation, ne reprenait pas l'intégralité des propositions formulées par la droite dans l'opposition', mais l'inspiration restait la même. Si le projet fut finalement abandonné, c'est pour des raisons conjoncturelles : le gouvernement, aux prises avec les manifestations étudiantes, battit en retraite ; mais, pour ne pas donner l'impression de renoncer purement et simplement, il mit en place une commission présidée par le vice-président du Conseil d'État et composée d'éminentes personnalités, dont le rapport servira plus tard de référence à la réforme de 19932

\section{Des "Sages" bien imprudents}

Cette commission s'est efforcée de donner aux débats un tour moins politique et plus théorique, partant du principe - logique à première vue, mais irréaliste, voire dangereux - que le code de la nationalité devait refléter les fondements de la solidarité nationale et contribuer à renforcer une identité nationale en perte de vitesse.

Parmi les personnes et les groupes auditionnés par la commission, il y avait ceux qui, tel le Club de l'Horloge, estimaient que la nationalité devait se transmettre exclusivement par la filiation, seule capable de préserver l'identité nationale, et qui déploraient que le code de la nationalité soit une machine à fabriquer des "Français de papier" qui ne se sentent pas vraiment français. Il y avait en face les promoteurs d'une théorie élective de la nation, débouchant logiquement sur la critique de l'automaticité et la défense du choix individuel exprimé par un acte positif ; cette analyse, défendue notamment par Alain Finkielkraut, a, de leur propre aveu, fortement influencé les membres de la commission. Il y avait enfin les tenants d'une conception objective et pragmatique de la nationalité, qui faisaient valoir qu'on est français avant tout par un phénomène d'acculturation lié à l'éducation reçue, notamment à l'école, et aux conditions de la vie quotidienne. Ceux-là récusaient, bien sûr, le "être français ça se mérite", mais aussi l'acte de volonté qui aboutirait à instaurer une discrimination entre les Français selon leur mode d'acquisition de la nationalité.

Entendant se situer à égale distance de la conception élitiste et de la conception pragmatique, la commission va opter finalement pour une sorte de "être français, ça se désire", partant de l'idée que le choix concrétisé par une manifestation de volonté serait un moyen de renforcer une identité nationale aujourd'hui menacée, et par là même de faciliter l'intégration des étrangers, jugée d'autant plus aisée que la conscience de l'identité nationale est plus forte. Moyennant quoi la commission proposait proposition qui sera reprise par la loi du 22 juillet 1993 - de passer du système en vigueur, dans lequel les jeunes nés en France deviennent français à leur majorité sans formalité sauf s'ils expriment le choix contraire, à un système dans lequel ils devraient, pour acquérir la nationalité française, en manifester explicitement la volonté entre 16 et 21 ans. Si le choix était fait entre 16 et 18 ans, aucun obstacle ne pourrail s'opposer à l'acquisition de la nationalité française ; au-delà de 18 ans, en revanche, certaines condamnations pénales empêcheraient cette acquisition.

Dans la foulée, la commission proposait aussi - et cette proposition sera comme la précédente entérinée par la loi du 22 juillet 1993 - que les parents ne puissent plus, pendant la minorité de leur enfant, demander pour lui la nationalité française par simple déclaration, ceci revenant à anticiper sur la manifestation de volonté de l'enfant qui serait ainsi privé du libre choix de sa nationalité. L'argument n'est guère sérieux : la volonté des enfants est sans doute une chose respectable, mais si l'on veut aller jusqu'au bout dans cette voie, c'est le principe même de l'autorité parentale qu'il convient de remettre en cause. En réalité, la considération déterminante pour les membres de la commission, comme elle le sera plus tard pour les parlementaires, était d'empêcher que les parents d'enfants nés en France ne soient incités à réclamer pour eux la nationalité française en vue de bénéficier de la protection accordée aux parents d'enfants français.

Le rapport de la commission reçut, dans l'ensemble, un accueil positif dans la presse et dans le munde politique. On sut gré à ces "Sages" d'être parvenus à dépolitiser en apparence le débat et à 


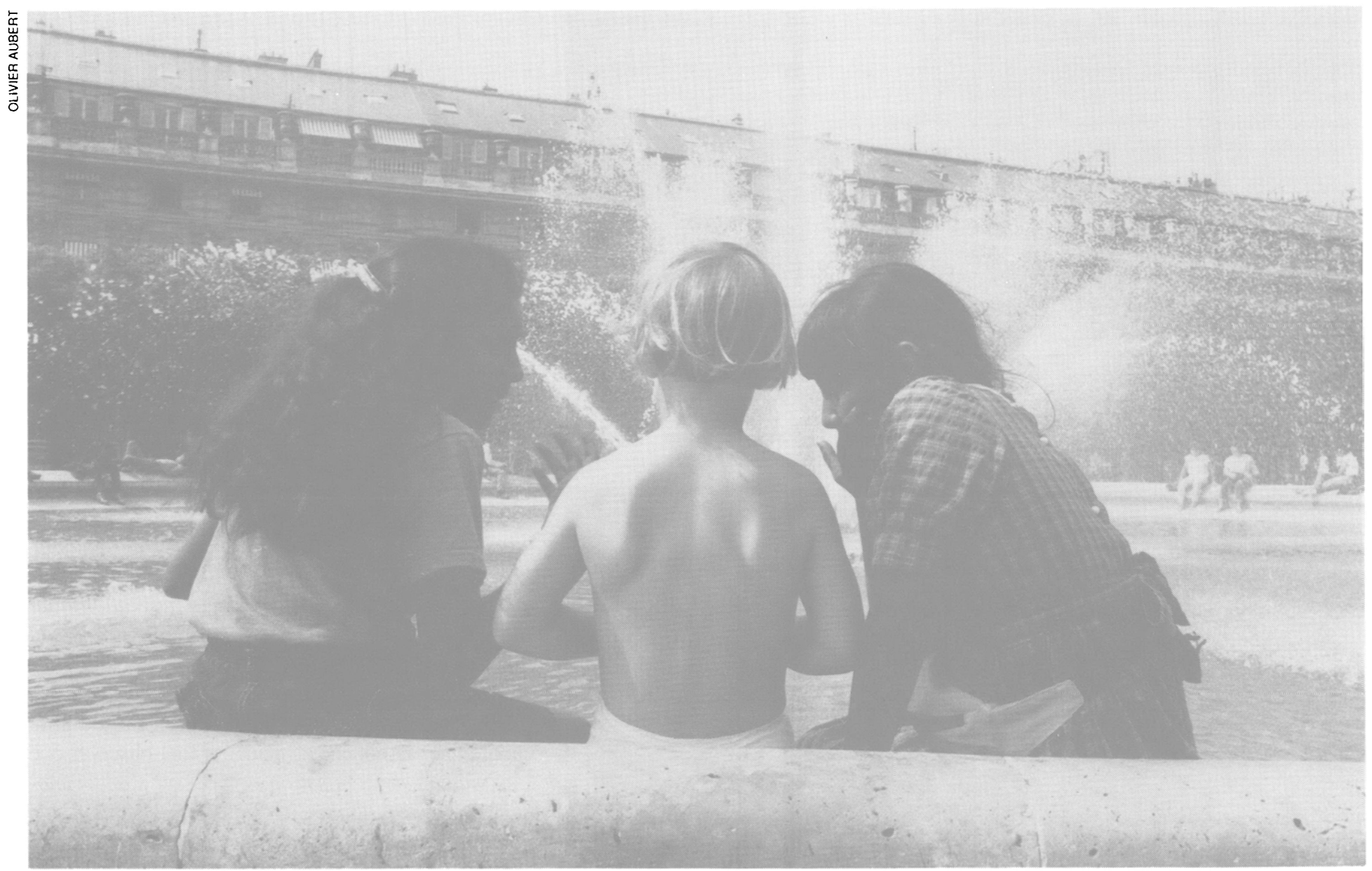

créer les conditions d'un consensus - gage de "vérité", comme chacun sait - entre des personnalités aussi différentes que Pierre Chaunu et Alain Touraine. Si des désaccords furent exprimés ici et là trop de place laissée au droit du sol, selon le Club de l'Horloge, pas assez de place selon SOS-Racisme qui plaidait pour le droit du sol intégral -, ils s'annulaient en quelque sorte aux yeux de l'opinion, confortant finalement l'idée que les propositions de la commission étaient justes, puisque se situant à égale distance des positions extrêmes.

Cet engouement politico-médiatique paralysa toute lecture véritablement critique du rapport de la commission. Pourtant, les postulats sur lesquels s'articulaient ses propositions étaient loin d'être parfaitement convaincants. Sur quoi la commission se fondait-elle pour affirmer que l'identité nationale était en voie d'affaiblissement? Et, surtout, par quel miracle l'acte de volonté exigé des quelques milliers de personnes postulant chaque année à la nationalité française contribuerait-il à résoudre les problèmes d'identité nationale auxquels la France était censée être confrontée ? Mystère...

Car si la conception élective de la nation mise en avant par la commission est incontestablement séduisante, comment ne pas remarquer que son seul effet concret sera de réclamer des jeunes nés en France, et d'eux seuls, un acte de volonté ? La référence à Renan, dont l'une des phrases les plus célèbres figure en exergue des propositions de la commission, est d'ailleurs des plus ambiguës. Car
Renan n'a pas seulement écrit : "L'existence d'une nation est un plébiscite de tous les jours"; il parle aussi de "la possession en commun d"un riche legs de souvenirs", de "la volonté de continuer à faire valoir l' héritage qu' on a reşu indivis", pour en arriver au constat que "la nation. comme l'individu, est l'aboutissement d'un long passé d'efforts, de sacrifices et de dévouements", que "le culte des ancêtres est de tous le plus légitime", car "les ancêtres nous ont fait ce que nous sommes" (Qu'est-ce qu' une nation? 1882). Présenter Renan comme le promoteur d'une conception ouverte de la nation est d'autant plus aventureux que l'auteur, on ne saurait l'oublier, en écrivant son texte, poursuivait une visée politique précise qui était de justifier l'appartenance à la France de l'Alsace-Lorraine en faisant prévaloir le critère de la volonté sur celui de la langue et de la culture.

\section{Le retour du refoulé}

En dépit du consensus recueilli par les propositions de la commission de la nationalité, ni la droite, pendant les quelques mois qui lui restent à gouverner, ni la gauche, entre 1988 et 1993 , ne prirent d'initiative pour les mettre en œuvre. À l'instigation de Charles Pasqua, le RPR fit cependant approuver sans débat par le Sénat une proposition de loi rédigée par Pierre Mazeaud qui mettait en forme législative les principales propositions de la commission : 


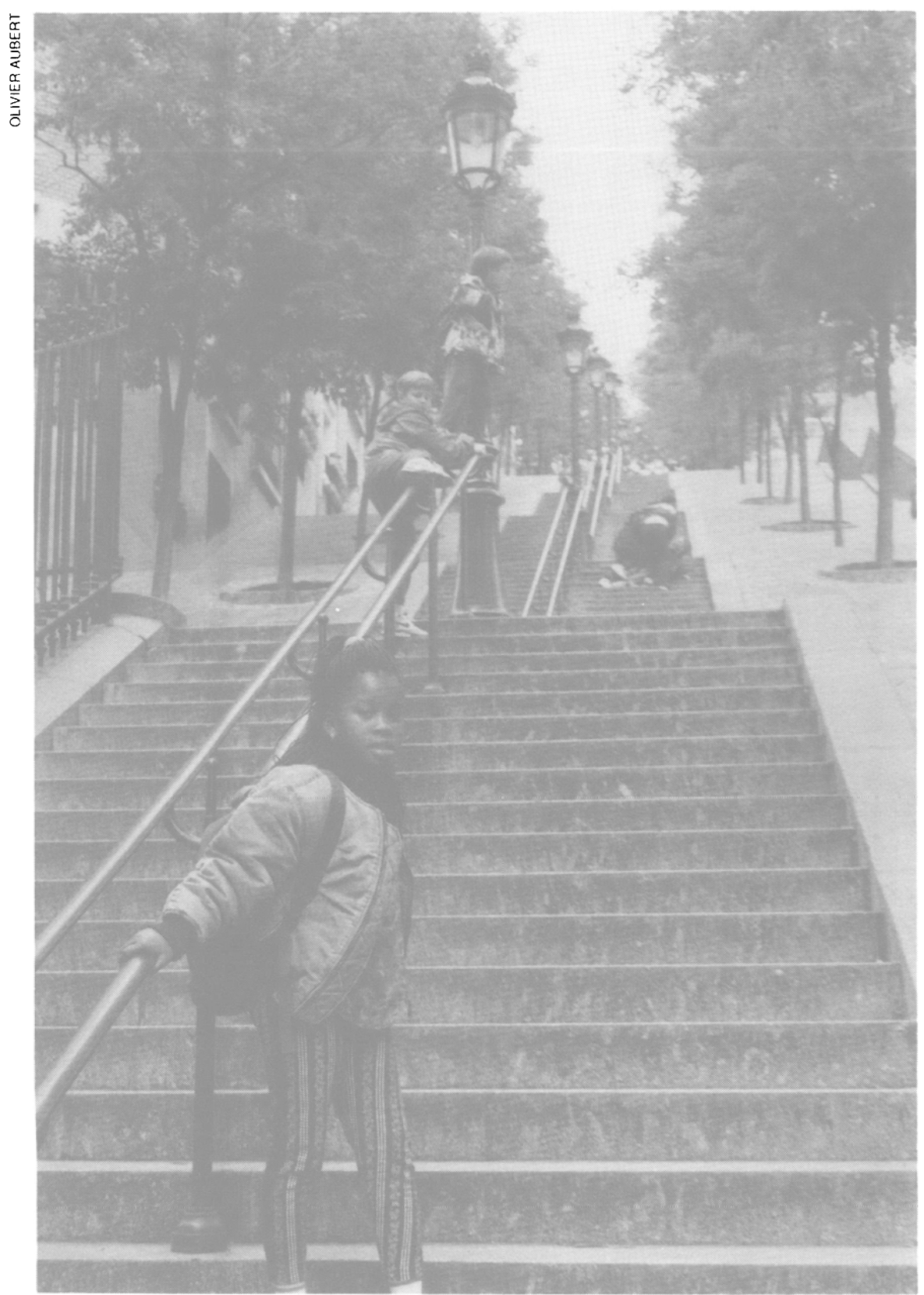

c'est ce qui permit au nouveau gouvernement, en 1993, de faire adopter très vite par le Parlement la proposition de loi déjà votée par le Sénat et qui deviendra la loi du 22 juillet 1993.

La lecture des débats parlementaires laisse claire-

3

Xavier de Villepin, Débats Sénat,

p. 1271.

Jacques Larché. Débats Sénat.

Pierre Mazeaud. Débats A. N. p. 347-348.

6

Christian Estrosi, ibid.. p. 401.

Pierre Louvot. Débats Sénat. p. 1280.

8

Nicole Catala. Débats A. N.. p. 402.

Xavier de Villepin. Débats Sénat. p. 1272. ment apercevoir les présupposés idéologiques qui animent députés et sénateurs, et les véritables motivations de la réforme.

La première de ces motivations, c'est l'état de l'opinion publique, car "si le législateur, pour prendre ses décisions, ne doit pas tenir compte que de l'opinion, il ne peut l'ignorer" et il doit "répondre de la manière la plus humaine à l'inquiétude de la population, causée par l'ampleur des mouvements de populations. ${ }^{3}$ ". La réforme vise donc - au minimum - à rassurer les Français.

En arrière-fond de la réforme, c'est bien, malgré les dénégations des uns et des autres, la question de l'immigration qui se profile au point d'en devenir obsédantc : l'amplification des flux migratoires et les difficultés de l'intégration font redouter à nos concitoyens "une dilution, une forme de délitement de la nationalité qui risque, à terme. de conduire à un dépérissement de l'idée même de nation"', et cela d'autant plus que l'immigration a changé de nature. Car - et ce constat revient lui aussi comme un leitmotiv - l'immigration d'aujourd'hui n'est plus celle d'hier. "Hier d'origine européenne, la population étrangère est aujourd' hui largement d' origine extraeuropéenne", et "l'intégration est d'autant plus difficile aujourd' hui que de plus en plus - nous devons le reconnaître - les étrangers qui vivent en France viennent de pays différents de ceux dont ils venaient dans le passés". "D'une immigration européenne proche culturellement, et donc facilement assimilable, nous sommes passés à une immigration dont l'intégration s'est révélée plus difficile en raison précisément de différences historiques et culturelles profondes 6 ."

Le constat revêt une tonalité plus inquiétante encore dans la bouche de ceux qui font observer que "les valeurs de civilisation ne peuvent s'accommoder, sans grave danger; des effets dissolvants d'une juxtaposition multiculturelle, génératrice de ghettos" et qu'à défaut d'agir "nous risquons d'assister à la progressive émergence d'une tour de Babel dont chacun sait qu' elle porte en elle les germes d'une confusion déstructurante ${ }^{7}$ ".

Cette inquiétude face à la menace qui pèse sur l'identité nationale, et la nécessité corrélative de trouver les moyens de la protéger sont évoquées par la plupart des intcrvenants. "La majorité [des Français] ne veut pas que notre pays se replie sur luimême, mais elle ne v'eut pas non plus d'une société émiettée, d'un puzzle de cultures, de confessions, de traditions qui, peu à peu, défigurent notre identité nationale. C'est donc à la sauvegarde de cette identité que le travail que nous avons entrepris doit tendres."

On attend donc de la réforme qu'elle renforce cette identité nationale menacée : "Le texte que nous examinons doit viser à l'affirmation d'une identité française forte et à une meilleure intégration $^{9}$."

Cette finalité attribuée au code de la nationalité renvoie à une conception ouvertement idéologique et non plus pragmatique du droit de la nationalité, qui se voit ainsi investi d'une charge symbolique importante. "Le droit de la nationalité est un droit dédié à ceux qui composent la nation, mais au-dessus d'eux à la nation elle-même. Sa finalité essentielle est de contribuer à la continuité de la nation, plus précisément à en maintenir l'unité. [Il] a pour fonction de consacrer l'appartenance des Français à la communauté nationale", énonçait l'exposé des motifs de la proposition de loi initiale. "La finalité du droit de la nationalité, c'est de contribuer à la continuité de la nation, plus précisément d'assurer, 
de maintenir sa propre unité. Il a pour fonction essentielle de consacrer l'appartenance des Français à la communauté nationale tout entière", dit à son tour le rapporteur à l'Assemblée nationale ${ }^{10}$, dont les propos seront repris à leur compte par les autres députés.

Dans ces conditions, la réforme du code de la nationalité est "avant tout porteuse d' un symbolell", "le symbole de l'indispensable réaffirmation de l'unité de la nation ${ }^{12}$." Mais au-delà de cette fonction symbolique, la réforme doit avoir pour objet de permettre de vérifier que l'appartenance à la collectivité nationale est fondée sur un véritable choix, et non sur des mécanismes automatiques. Assurer la pérennité de la nation française "ne saurait être plus longtemps compatible avec les mécanismes d'acquisition automatique par lesquels un grand nombre de gens se trouvent français sans l'avoir voulu13". Les modifications juridiques proposées visent donc à faire "respecter la règle selon laquelle la nation n'existe que par l'adhésion formelle de ses membres ${ }^{14}$ ", conformément à la conception française de la nation dont "on sait bien, depuis Renan, qu'elle est avant tout élective. [non pas] fondée sur. la race ou l'ethnie mais sur le libre consentement des personnes ${ }^{15}$ ".

Loin de remettre en cause "les principes qui font de notre droit de la nationalité [...] un modèle spécifique, hérité de notre histoire et d'une conception élective de la nation ${ }^{16}$ ", la réforme proposée tend au contraire à leur donner leur plein effet. Car aujourd'hui "notre droit du sol méconnaît cette idée fondamentale que la nation repose sur des valeurs communes, sur une acceptation réciproque et sur un vouloir vive ensemble ${ }^{17}$ ".

L'invocation obsessionnelle de Renan ne saurait masquer que, plus que la fidélité à des principes, c'est la méfiance qui, chez bon nombre de parlementaires, conduit à subordonner l'acquisition de la nationalité française à une manifestation explicite de volonté. S'il faut vérifier qu'ils veulent être français, c'est parce qu'on ne peut pas le postuler, et si l'on ne peut pas le postuler, c'est parce que les étrangers nés en France n'appartiennent plus désormais à la culture européenne : la boucle est ainsi bouclée...

Implicitement, et parfois même explicitement, se profile aussi dans certains discours l'idée que le droit du sol ne donne pas les mêmes garanties d'appartenance à la nation que le droit du sang : "Maintenir cette identité, maintenir aussi la cohésion de la communauté nationale, cela postule aujourd' hui que soit mieux assurée, mieux vérifiée la volonté d'être français chez ceux qui ne le sont pas par le sang18." Cette méfiance envers le droit du sol est également exprimée par Philippe de Villiers, pour qui le système le plus cohérent et le plus simple consisterait à retenir la filiation d'un côté et de l'autre la naturalisation par décret individuel, puisque cette dernière procédure est "celle qui permet de vérifier le mieux que le postulant est bien volontaire, qu'il est "digne" au sens de l'article 46, et qu'il est assimilé, c'est-à-dire qu'il partage l'ensemble de nos valeurs, la connaissance de notre histoire, de notre langue et le respect de nos institutions ${ }^{19}$ ".

Devenir français, au demeurant, est un honneur, et l'on voit mal dès lors en quoi l'exigence d'un acte de volonté explicite pourrait apparaître comme vexatoire pour les intéressés. "Le système par récépissé nous paraît un peu réducteur par rapport à l'honneur d'intégrer la nation française", remarque l'un20. "Est-il si dérisoire de devenir français que l'acquisition de notre nationalité ne mériterait même pas d'accomplir la plus simple des formalités administratives, par exemple la demande d'une carte d'identité ?", s'interroge l'autre21. Et en définitive, la réforme permettra "de mieux intégrer les étrangers appelés dans la dignité à devenir français, par une démarche éclairée?2".

En exigeant des personnes nées en France de parents étrangers une manifestation positive de volonté, les promoteurs de la réforme poursuivent donc simultanément deux objectifs : mettre une barrière - essentiellement symbolique, certes, mais barrière quand même - à l'accès à la nationalité française, et rassurer une opinion présumée inquiète pour l'identité nationale.

Mais on ne manipule pas impunément les symboles : l'identité nationale a servi d'alibi à une réforme qui, en dépit du caractère apparemment limité de ses conséquences concrètes, porte la marque de son inspiration initiale faite de crainte et de suspicion, et qui instaure une démarcation plus visible entre les Français de naissance, généralement Français par filiation, donc par le "sang", et ceux qui, potentiellement Français par le droit du sol, auront désormais le "choix" de ne pas le devenir.

\section{Le rétrécissement de l'accès à la nationalité française}

En dépit de la volonté des auteurs de la réforme d'en minimiser la portée, la loi du 22 juillet 1993 apporte à la législation en vigueur des modifications importantes qui ont presque toutes pour objet de rendre plus difficile l'accès à la nationalité française. L'essentiel des changements portent sur les effets du jus soli et sur l'acquisition de la nationalité française par mariage, tandis que, de façon significative, l'attribution de la nationalité française par filiation n'est pas concernée par la réforme.
Pierre Mazeaud, Débats A.N. p. 345 .

11

Ernest Cartigny, Déhats Sénat. p. 1283.

12

Jacques Larché, ibid., p. 1264. 13

Ernest Chénière. Débats A. N.. p. 359.

14

Pierre Mazeaud. ibid.. p. 346.

15

Pierre Méhaignerie, ibid.. p. 352.

16

Jean-Jacques Hyest, ibid., p. 387.

17

Philippe de Villiers. ibid., p. 410. 18

Nicole Catala. ibid.. p. 402.

19

Philippe de Villiers, ibid., p. 410. 20

Francis Delattre. ibid.. p. 360.

21

Henri Cuq. ibid.. p. 390

22

Pierre Louvot. Débats Sénat, p. 1280 . 


\section{Le droit du sol}

S'il n'est pas remis en cause dans son principe, ses effets sont néanmoins sensiblement restreints.

L'enfant né en France de parents étrangers, qui devenait français sans formalité à l'âge de 18 ans s'il avait eu sa résidence en France pendant les cinq années qui précèdent, devra désormais manifester sa volonté de devenir français entre 16 et 21 ans, tandis que ses parents ne pourront plus non plus réclamer pour lui la nationalité française par simple déclaration au cours de sa minorité.

\section{La réforme instaure une démarca- tion entre les Français par le "sang" et ceux potentiellement français par le droit du sol}

Si le jeune manifeste sa volonté de devenir français entre 16 et 18 ans, le système paraît extrêmement libéral, puisque d'une part aucun obstacle ne peut s'opposer à l'acquisition de la nationalité française et que d'autre part, même mineur, il n'a pas besoin de l'autorisation de ses parents. Il n'en est pas moins illusoire de penser que son choix sera entièrement libre : d'abord, parce qu'un adolescent ne s'affranchit pas si aisément de l'influence de sa famille - et s'il y parvient ce peut être au prix d'un conflit qu'il n'était pas forcément opportun de susciter ; ensuite, parce que s'il décide d'accomplir les formalités lui permettant de devenir français, on voit mal comment, en pratique, il pourra se passer de l'aide de ses parents pour se procurer les pièces nécessaires.

Si la demande est formulée après l'âge de 18 ans, l'acquisition de la nationalité française n'est plus automatique : certaines condamnations pénales pour des faits commis après la majorité, ou encore l'existence d'une mesure d'expulsion ou d'interdiction du territoire font perdre au jeune étranger le droit de devenir français. Les infractions qui font obstacle à l'acquisition de la nationalité française sont d'une gravité inégale ; mais c'est surtout l'interdiction du territoire qui risque en pratique de représenter l'obstacle le plus important, notamment parce que, parmi les délits susceptibles d'entrainer une interdiction du territoire figurent "le transport, la détention, l'offre. la cession, l'acquisition ou l'emploi illicite de stupéfiants", incriminations dont on sait qu'en pratique elles visent au même titre les petits dealers occasionnels et les véritables trafiquants.
En pratique, il est probable qu'un certain nombre des jeunes concernés, pour des raisons diverses manque d'information, négligence, révolte, pressions familiales... -, n'accompliront pas la démarche volontaire exigée d'eux et resteront étrangers dans le pays où ils vont pourtant vivre. On peut craindre en particulier que les jeunes qui ne sont plus scolarisés sans être pour autant insérés professionnellement soient mal informés de leurs droits, et qu'ils aient de surcroît des difficultés à faire la preuve qu'ils résidaient en France au cours des cinq années précédant leur demande.

On peut surtout redouter les effets déstabilisateurs de la nouvelle procédure pour ces jeunes que l'on va mettre en demeure - à un âge et dans un contexte peu propices à des décisions sereines - de dire s'ils veulent devenir français, et de faire un choix que leurs camarades nés de parents français n'ont pas à faire. Avec comme risque supplémentaire - et les premiers échos qui parviennent des lycées montrent que ce risque n'est pas imaginaire - de favoriser l'apparition d'une fracture entre les jeunes d'une même génération en fonction de leur origine.

Par ailleurs, en supprimant la faculté de réclamer pour l'enfant mineur la nationalité française par simple déclaration, et en différant dans tous les cas jusqu'à 16 ans au moins l'accès à la nationalité française des jeunes nés en France de parents étrangers, on empêchera sans doute certains adultes d'obtenir plus aisćment unc cartc de résident ; mais on rendra surtout la vie plus compliquée à des milliers d'enfants dont l'avenir est en France, dont le législateur lui-même reconnaît qu'ils sont des Français en puissance, et qui ont donc intérêt, pour des raisons à la fois matérielles, juridiques et psychologiques, à ce que leur nationalité soit fixée le plus tôt possible.

\section{Le"double droit du sol"}

Le double droit du sol, en vertu duquel est Français de naissance l'enfant né en France dont l'un des parents est lui-même né en France, n'est pas non plus remis en cause dans son principe ; mais son champ d'application est considérablement limité.

En premier lieu, la naissance dans un territoire qui avait le statut de colonie française ou de territoire d' outre-mer ne sera plus désormais assimilée à la naissance en France. Sur ce point encore, le législateur s'est conformé aux propositions de la commission, qui faisait valoir que cette disposition était anachronique et qu'en la supprimant on ne ferait que tirer toutes les conséquences de la décolonisation. Derrière l'argument de l'anachronisme se cache en réalité la volonté de faire obstaclc à l'installation en France des ressortissants africains qui peuvent invo- 


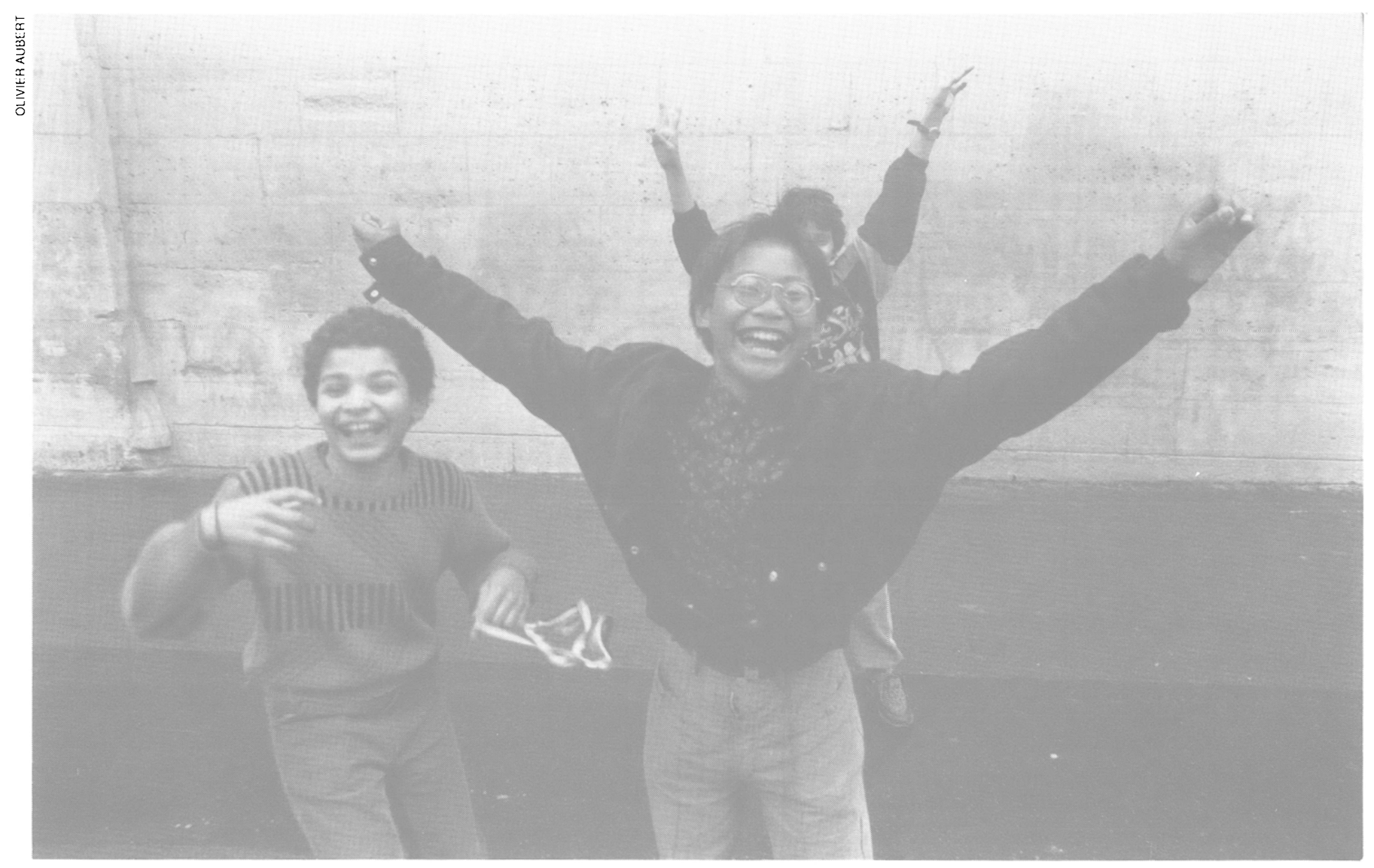

quer leur qualité de parents d'enfants français pour obtenir une carte de résident.

- En second lieu, les enfants nés en France de parents nés en Algérie avant l'indépendance, donc dans un département français, ne bénéficieront du double droit du sol que si, au moment de leur naissance, l'un au moins de leurs parents résidait régulièrement en France depuis cinq ans. Le but de cette disposition, qui ne figurait pas dans la proposition de loi initiale et a été introduite au cours des débats parlementaires, est clair : il s'agit là encore de faire obstacle à l'établissement durable en France de ressortissants algériens qui pourraient se prévaloir dans l'immédiat ou plus tard de leur qualité de parents d'enfants français. On a complaisamment évoqué, lors de la discussion du projet, pour alimenter la thématique de la fraude, et comme si elles étaient des milliers à pouvoir le faire, les femmes algériennes qui viennent accoucher en France dans le seul but de conférer la nationalité française à leur enfant. Mais on a oublié de rappeler que les ressortissants algériens, contrairement aux ressortissants étrangers d'autres nationalités, n'ont pas droit, en tant que parents d'enfants français, à l'obtention de plein droit d'un titre de séjour, ce qui limite singulièrement l'intérêt de fraude alléguée.

La nouvelle disposition apparaît donc surtout comme une vexation et une marque de suspicion supplémentaire à l'égard des Algériens. Elle risque de surcroît de créer des problèmes insurmontables aux enfants concernés lorsque, avant de leur délivrer un certificat de nationalité, on leur demandera de prouver que leurs parents résidaient en France depuis plus de cinq ans sous couvert d'un titre de séjour régulier au moment de leur naissance.

\section{Les conjoints de Français}

Ils ne pourront plus acquérir la nationalité française par déclaration qu'au bout de deux ans et non plus six mois de mariage et de vie commune, ce délai étant toutefois supprimé si le couple a des enfants. La cessation de la communauté de vie entre les époux dans l'année qui suit l'enregistrement de la déclaration - alors que le mariage aura été célébré plus de trois, voire quatre ans auparavant ! - constituera de plus une présomption de fraude permettant d'annuler la déclaration. Cette modification n'est que l'une des multiples manifestations de la suspicion qui pèse par principe sur les mariages entre Français et étrangers et dont on retrouve également la trace dans la loi du 24 août 1993 sur l'entrée et le séjour des étrangers en France.

Au total, c'est bien à un rétrécissement de toutes les voies d'accès à la nationalité française, filiation exclue, que la réforme aboutit, reflétant bien le climat général de crainte et de suspicion dans lequel le projet a pris naissance, a mûri, et a finalement abouti. Mais au-delà de ses effets concrets, c'est la justification idéologique de la réforme qui est la plus inquiétante. 